\title{
Accuracy of MRI in Rectal Cancer After Preoperative Neoadjuvant Chemoradiotherapy
}

\author{
Saeed Naghibi ${ }^{1}$ \\ ${ }^{1}$ Islamic Azad University
}

Received 2016 December 21; Accepted 2017 February 08.

\begin{abstract}
Background: About 1/4 colorectal cancers are related to the rectum. There 40.000 patients with the mean age of 50 years diagnosed with rectal cancer in USA. The symptoms are in a wide spectrum such as rectal bleeding, changing of defecation habits, pain, incontinenecy in the case of esphincter invasion. The diagnosis depends on getting a detailed history and a physical examination. For years the main treatment of rectal cancer was abdominoperineal resection with permanent clostomy. Today's treatment plan includes less invasive surgical techniques combined with neoadjuvant chemoradiotherapy which results in less recurrent rates. MRI is a reliable modality in staging primary tumors but there has been conflicts about its accuracy for restaging after neoadjuvant chemoradiotherapy. In this study, accuracy, sensitivity and specifity of MRI will be discussed.

Methods: Study on 34 patients diagnosed with rectal adenocarcinoma referred to oncology clinic was conducted. The treatment plan included 4050 cGY radiotherapy and 5FU, Xeloda chemotherapy. All patients were assessed with 1/5 T MRI 6 to 8 weeks after treatment. T1 and T2 images with sagittal, cronal and axial planes with and without contrast agent were obtained and interpreted by two radiologists and compared with pathologic specimens as a gold standard.

Results: The demographic information was: mean age of 65/26 years, 19 men (55/9\%), 15 women (44/1\%). Accuracy of MRI at T and N staging were $38 / 2 \%$ and $61 / 8 \%$ with the highest sensitivity of T3NM0 and specifity of T1N2.

Conclusions: MRI can be used for assessing rectal tumors and restaging after preoperative chemoradiotherapy.
\end{abstract}

Keywords: MRI-Rectal, Cancer-Neoadjuvant, Radiochemotherapy

This is an abstract presented in the 33rd Iranian congress of radiology (ICR) and the 15th congress of Iranian radiographic science association (IRSA) 